\title{
A SERVEY ON WIRELESS MESH NETWORKING MODULE
}

\author{
Mamta Nakhate 1 , Abhay Satmohankar ${ }^{2}$ \\ ${ }^{I}$ M.Tech Scholar, Department of Electronics Engineering. Wainganga collage of Engineering, Nagpur, India. \\ ${ }^{2}$ Assistant Professor, Department of Electronics and Telecommunication Engineering, WCEM, Nagpur, India.
}

\begin{abstract}
There is a rapid development in sensor devices as well as increase in communication range with various low power wireless radio transmitter and receiver using wireless mesh network. Wireless mesh networking is useful to collect the information from different sensors that are widely distributed over a large area. In this survey, we mainly focus on open source module that is shown by various authors. For this purpose many authors use Zigbee module for the development of sensor systems with capability of mesh networking. In this survey, we mainly focus on wireless mesh networking, for that we studied different wireless sensors to transmit and receive the data from them and can monitor the output at regular interval on our screen. WSN consist of different sensors that are distributed with the capabilities of computing, processing and communication can continuouslysense the data in terms of packets and transmit that data at regular interval. They design such model in which integrates the various functions such as type of network, data routing and scheduled of transmission data. Also design wireless mesh network structure for various monitoring application such as industrial, agricultural, medical and so many with a good average packet delivery ratio upto 93\%. This proposed system has the advantage of low cost combined with high reliable information of transmitter without complications of wireless mesh networking. And finally we conclude from that study of WMN, the range of communication can be increased by Using Pro-Zigbee module (up to 1.6Km) as well as we can monitor that, if one of the node is fail to transmit a data,then their data will automatically stored in neighboring node as backup.
\end{abstract}

Keywords: Wireless Sensor Network, Wireless Mesh Network, Different Sensors, Zigbee Module.

\section{INTRODUCTION}

The wireless mesh networking is used to monitor the environmental issues. It has the advantage of imposing no issues due to cabling of data transmission. Wireless mesh network consist of more than one node and it has combined multiple transceiver to co-operatively transmit and depend their data thereby the range of communication can be extended.

The WSN generally consist of large number of low cost and power multi functional sensor nodes. In Wireless sensor network, the different nodes are communicated with each other wirelessly over a short distance and those capable of organizing themselves in an autonomous multi hop network. WSN system also has been used in many applications such as, health monitoring, environmental monitoring and agricultural monitoring. Wireless sensor network unable for environment monitoring of large areas with so many wireless sensors. Therefore in many of the paper they combined the characteristics of WSN for the formation of mesh network. In WSN, there is sink as master node and multiple slave nodes are connected with each other for the formation of mesh network.

In this we described, combines various nodes and form a network that regularly sense and may control after monitoring data. The main objective of developing such system, that formation of wireless mesh network design, and adjust the topology according to the changes of radio interference obstacles, transfers the various sensors data back to the sink and upload the data from sensors at regular intervals. We design WMN module, which can be integrate easily with sensor system for extending the communication range as well as reduce the interference between them.

In this module we used Pro Zigbee model, it is one of the most popular design which supports wireless mesh networking module which is based on the IEEE standard 802.15.4. Zigbee is used as the wireless communication unit with various sensor nodes and Pro-Zigbee protocol is selected because it has low cost and low power wireless communication protocol.

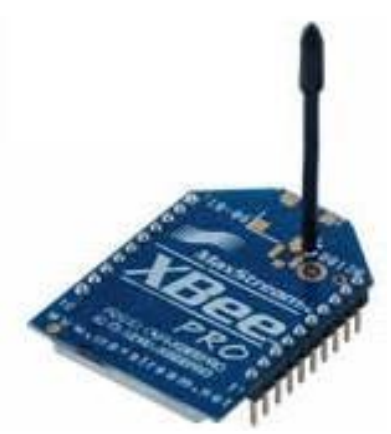

Fig-1: RF module of PRO-ZIGBEE

The silent feature of Pro Zigbee module is as follows

- Power output is $63 \mathrm{~mW}$

- Indoor Range is upto 90meters

- Outdoor range/Line of sight range is upto $1.6 \mathrm{Km}$ 
- RF data rates is upto $250 \mathrm{Kbps}$

- Interface data rate is upto $115.2 \mathrm{Kbps}$

- Operating frequency is $2.4 \mathrm{Ghz}$, because operating on ISM band

- Receiver sensitivity is $-100 \mathrm{dBm}$.

They design wireless module which are easy to configure with system and it's suitable for point to point, point to multipoint network and also compatible with mesh network. The various sensor nodes in WSN consist, main processor, data aquaization unit, memory unit to stored the data and different sensors.

In our paper, we use one master node and three slave nodes for the different monitoring application such as agricultural, industry and monitor power of any type of circuit. For that purpose we use ATMEGA16 processor for master node and for slave node we use ATMEGA8 processor. We can collect the data at any instant of time from any nodes and that data can easily stored in main processing unit. In survey we can clearly seen that many authors, worked on open source module which is compatible for both hardware and software. But we can also be used to study the performance of WMN on real time base parameters and that we are shown in future scope.

\subsection{ARCHITECTURE OF WMN}

In wireless mesh networking we use various sensor nodes for the monitoring of various application such as industrial, agricultural and power measurements of given that means we can measure the current and voltage. The WMN module can design using 8 bit controller based on AVR enhanced RISC architecture, ATMEGA16 is low power CMOS 8 bit controller with low cost RF transmitter and receiver circuit in which output power is nearly equal to $1 \mathrm{~mW}$ at $2.4 \mathrm{GHz}$.

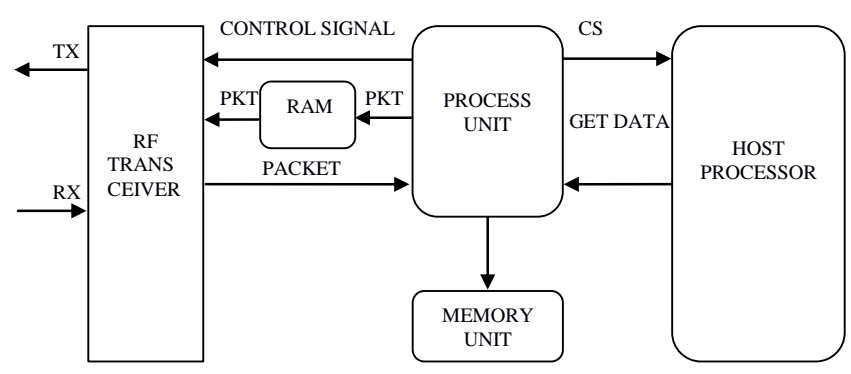

Fig-2: Functional diagram of WMN

The primary stage to design such model is connecting ATMEGA16 to main processor and that main processor interface the control signal and transfers the data in terms of packets to main processor. In this, the main processor only deals with ATMEGA16 8 bit microcontroller whenever the data packets send or receive. The main processor does not need to worry about the various nodes that are connected through this controller.

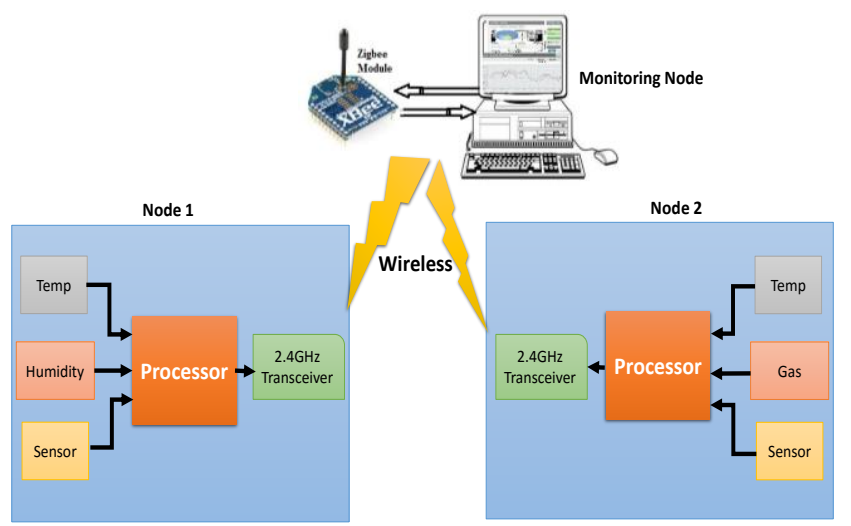

Fig-3: Basic structure of WMN

\subsection{DESIGN OF WSN MODULE}

A WSN is the combination of, various hardware sensors, combines sensing, computation and communication for monitoring and recording conditions at diverse location. Wireless network consist of various sensor nodes which are used to detect or sense the data. The parameters that are commonly and regularly monitored such as temperature, humidity, pressure, sound intensity, gas, voltage, power, soil and many other applications.

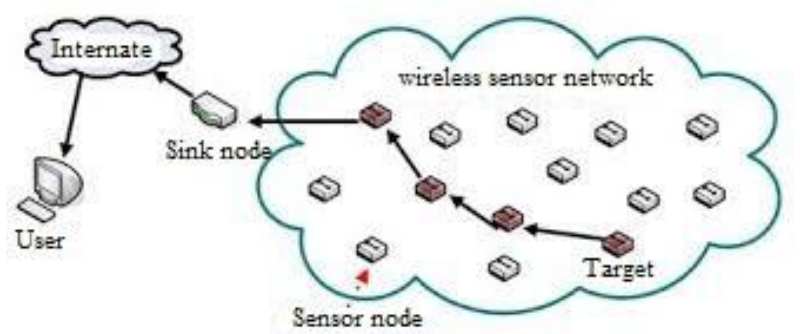

Fig-4: Structure of Wireless sensor network

A WSN consists of multiple stations in given region called as sensor nodes. The size of sensor node is very small, lightweight and it's portable. The sensor nodes are connected to the user through internet and the master node

\subsection{TRANSMITTER NODE}

There are three different nodes we are used in this proposed system as transmitter nodes for different application such as industry, agriculture and power saving circuit. And the final structure of one of the node is as shown below.

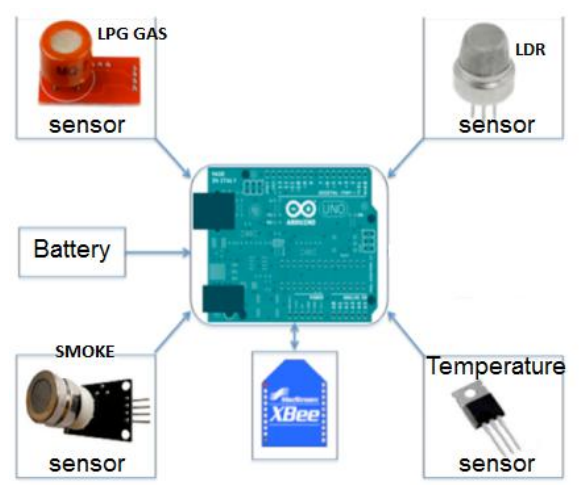

Fig-5: Example of sensor node for industrial application 
Each transmitting nodes consist of one ATMEGA 8 microcontroller with Zigbee wireless module and various sensors such as temperature sensor, Gas sensor, smoke detector sensor and LDR sensor.

\subsection{FORMATION OF MESH NETWORK}

In our proposed system, we use total four nodes including master node for various applications. Each sensor node of the system having identification number assigned to it. And each nodes has corresponding timeslot in every TDMA cycle, to avoid the different sensor nodes try to transmit the data at same timeslot. When the formation of mesh network, use master node as Sink node and other node act as sensor node. Master node assigned the unique identificatiob number as ID 0 functioned as Sink. And the other nodes which identificatib number is not zero are sensor nodes which sense the data and transfer to sink node.

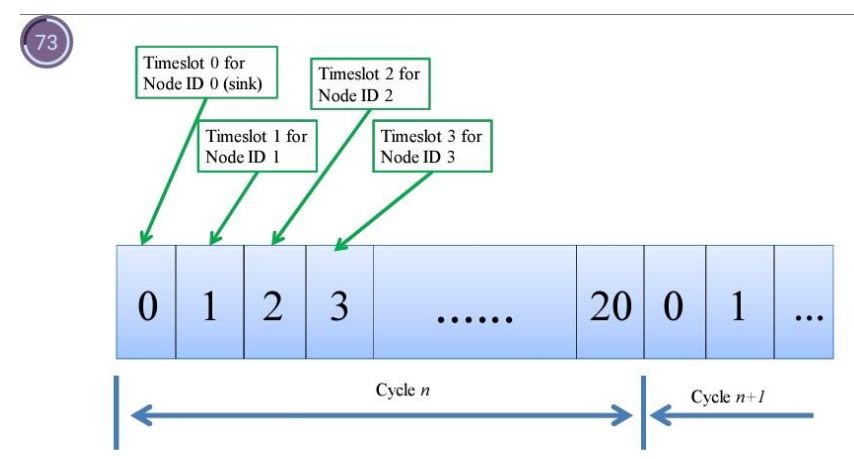

Figure-6: TDMA structure for different slot

\section{LITERATURE SURVEY}

1) Hsiao-Hsien Lin1, Hsi-Yuan Tsai1, TengChieh Chan1, Yen-Shuo Huang1, Yuan Sun Chu1, Yu Chieh Chen2

“An Open-Source Wireless Mesh Networking Module for Environmental Monitoring[1]" 978-1-4799-6144-6/15/2015 IEEE

- In this paper, They design such model in which integrates the various functions such as type of network, data routing and scheduled of transmission data..

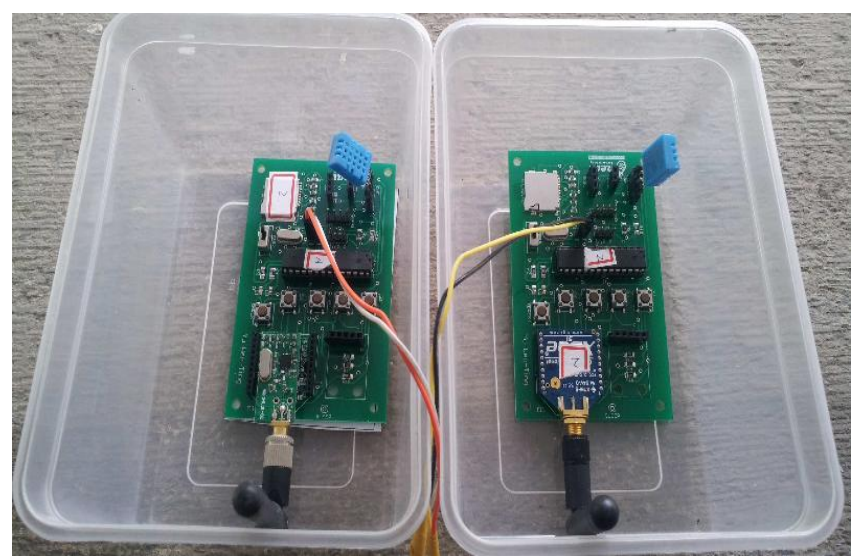

Fig-7: Two version of WSN proposed based on WMN module
- They design mesh network for 10 node with average packet delivery ratio is $95.58 \%$.

- They implement different various module and compare the results of two different module.

2) Ashenafi Lambebo, Sasan Haghani, Department of Electrical and computer Engineering, University of District of Columbia, 4200 Connecticut Ave N.W., Building 42.[2] Wshingtion DC, 20008[2]

"A WSN for Envirnmental Monitoring of Greenhouse Gases"

- In this paper, they implement WSN for real time and continuous environmental monitoring of greenhouse gases [2].

- They used tree topology as well as WSN network consisting two sensor nodes and one base station [2].

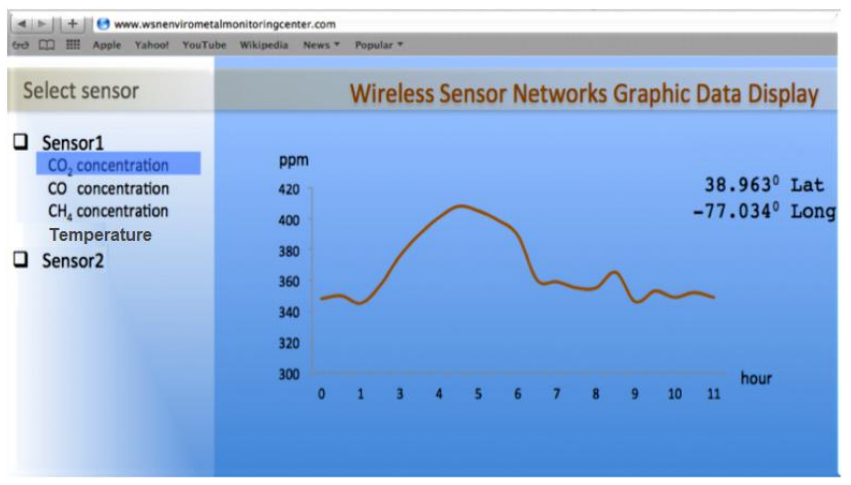

Fig-8: Output result of Gas sensors

-They tested using open source and inexpensive hardware to measure the concentration level of several greenhouse gases[2].

3) B.P. Ladgaokar and A.M. Pawar, IJAET[5], July 2011, ISSN: 2231-1963

"Design and implementation of sensor node for wireless sensors network to monitor humidity of high tech polyhouse environment.[5]"

- In this paper they monitor the environmental parameters of high tech polyhouse the wireless sensor network are developed[5].

- Based on embedded technology and RF module Zigbee wireless sensor node is designed about highly promising AVR ATMEGA 8L microcontroller and implemented for WSN development[5].

4) Akshay D. Deshmukh, Dr. Ulhas B. Shinde, Krishna M. Biradar, IJIERT,ICITDCEME15. Conference proceedings ISSN No. 2394-3696

"A review: Environmental monitoring applications using wireless sensor technology." 
- In this paper WSN system is proposed with open source hardware and software platform.

- For this purpose they used Raspberry Pi microcomputer which act as base station, Zigbee and graphical user interface for application.

5) Stefan Bouckaert, Eli De Poorter, Pieter De Mil, Ingrid Moerman, Piet Demeester

"Interconnecting Wireless Sensor and Wireless Mesh Networks: Challenges and Strategies"

IEEE "GLOBECOM"2009."

- In this paper, they shown that how WSN network can be combined with wireless mesh networks to obtain optimized solution.

- The wireless mesh network can be used to connect separate sensor networks, to connect various WSN sensor nodes with a monoting platfoem.

\section{CONCLUSION}

There are so many papers on environmental monitoring using wireless sensor network. But in this paper we used more than one application for monitoring. We make mesh module in that different wireless sensors are combinly used to transmit the data to main node at every time without fail. We not only monitored the different fields but also control them if they cross the threshold level of particular sensor. For that purpose we provide a relay and buzzer, which indicates threshold level crossed and we provide relay in each system to control the application. If any of the node is fail in mesh topology then their data is stored as backup to its neighboring node.

So according to this we can monitor environmental application as well as control the appliances that are connected as sensor.

\section{REFERENCES}

[1] Hsiao-Hsien Lin1, Hsi-Yuan Tsai1, Teng-Chieh Chan1, Yen-Shuo Huang1, Yuan-Sun Chu1, Yu-Chieh Chen2, TaiShan Liao2, Yao-Min Fang3, Bing-Jean Lee3, Huang-Chen Lee1*. "An Open-Source Wireless Mesh Networking Module for Environmental Monitoring" 978-1-4799-6144$6 / 15 / \$ 31.00$ C2015 IEEE

[2] Gutierrez, J.; Villa-Medina, J.F.; Nieto-Garibay, A; Porta-Gandara, M.A, "Automated Irrigation System Using a Wireless Sensor Network and GPRS Module," Instrumentation

and Measurement, IEEE Transactions on , vol.63, no.1, pp.166,176, Jan. 2014.

[3] Ashenafi Lambebo, Sasan Haghani

Department of Electrical and Computer Engineering University of the District of Columbia 4200 Connecticut Ave N.W., Building 42 Washington DC, 20008. "A Wireless Sensor Network for Environmental Monitoring of
Greenhouse Gases" ASEE 2014 Zone I Conference, April 35, 2014, University of Bridgeport, Bridgpeort, CT, USA.

[4] Araujo, A; Garcia-Palacios, J.; Blesa, J.; Tirado, F.; Romero, E.; Samartin, A; Nieto-Taladriz, O., "Wireless Measurement System for Structural Health Monitoring With High Time-Synchronization Accuracy," Instrumentation and Measurement, IEEE Transactions on , vol.61, no.3, pp.801,810,

March 2012.

[5] B. P. Ladgaonkar and A. M. Pawar

Embedded Technology and Instrumentation Laboratory Post Graduate Department of Electronics, Shankarrao Mohite Mahavidyalaya. "DESIGN AND IMPLEMENTATION OF SENSOR NODE FOR

WIRELESS SENSORS NETWORK TO MONITOR HUMIDITY OF HIGH-TECH POLYHOUSE ENVIRONMENT" International Journal of Advances in Engineering \& Technology, July 2011.@IJAET ISSN: 22311963.

[6] ZigBee Specification, ZigBee Alliance Std. 2005 [online]. Available at : http://www.zigbee.com

[7] S. Kim, D. Culler and J. Dammel, (2007) "Structural Health monitoring using wireless sensor network", IPSN

[8] N. Xu, "A survey of sensor networks applications", http://eln.usc.edu/ningxu/papers/survey.pdf.

[9]. Z. Eswawan, F. Ahmad (2005) “Wireless sensor network based system for fire endangered Areas",

ICITA, 2 203-207.

[10] K. K. Chintapaludi, ,(2006) “Design of Wieless sensor network based structural health monitoring

system" University of Southern California, Los Angeles CA USA ISBN 978-0-542-87355-3.

[11] A. Das and R. Bag, (2010) "Wireless sensor Network Based monitoring systems: A review and State

of Art Applications", Int. J. Comp, Appl. Engg. Tech and Sc. 31142 .

[12] Araujo, A; Garcia-Palacios, J.; Blesa, J.; Tirado, F.; Romero, E.; Samartin, A; Nieto-Taladriz, O., "Wireless Measurement System for Structural Health Monitoring With High Time-Synchronization Accuracy," Instrumentation and Measurement, IEEE Transactions on , vol.61, no.3, pp.801,810, March 2012

[13] Huang-Chen Lee; Yao-Min Fang; Bing-Jean Lee; Chung-Ta King, "The Tube: A Rapidly Deployable Wireless Sensor

Platform for Supervising Pollution of Emergency Work," Instrumentation and Measurement, IEEE Transactions on , vol.61, no.10, pp.2776,2786, Oct. 2012

[14] Gutierrez, J.; Villa-Medina, J.F.; Nieto-Garibay, A; Porta-Gandara, M.A, "Automated Irrigation System Using a Wireless Sensor Network and GPRS Module," Instrumentation and Measurement, IEEE Transactions on , vol.63, no.1, pp.166,176, Jan. 2014 


\section{BIOGRAPHIES}

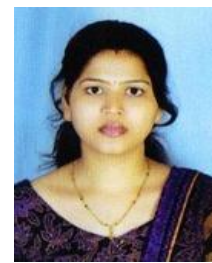

Miss. Mamta Nakhate, B.E. in Electronics Engineering(2009),Nagpur. Student of M.Tech $4^{\text {th }}$ Semester (Electronics Engg.), Wainganga College Of Engineering \& Management. Nagpur.

Mail id:- mamtanakhate@yahoo.in

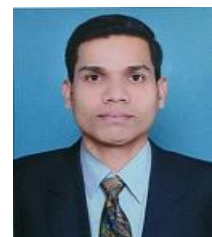

Prof. Abhay Satmohankar, M.Tech in Communication Electronics(2013), Nagpur B.E. in Electronics and communication (2008), Nagpur Faculty of Electronics and Telecommunication department, Wainganga College of Engineering and Management, Nagpur.

Mail id :- abhaysatmohankar01jun@gmail.com 